\title{
Clear cell adenocarcinoma of the uterine cervix in a 24-year-old woman. Case report and review of the literature
}

\author{
Leszek Gottwald², Jerzy Korczyński², Ewa Góra³, Renata Kusińska4, Ewa Rogowska \\ Katarzyna Wójcik-Krowiranda ${ }^{6}$, Andrzej Bieńkiewicz ${ }^{6}$
}

\author{
1Department of Palliative Medicine, Regional Cancer Center, Copernicus Memorial \\ Hospital of Lodz, Poland \\ 2Department of Fetal Medicine and Gynecology, I Chair of Obstetrics and Gynecology, \\ Medical University of Lodz, Poland \\ ${ }^{3}$ Department of Perinatology and Gynecology, Polish Mother's Memorial Hospital \\ of Lodz, Poland \\ ${ }^{4}$ Department of Pathology, Chair of Oncology, Medical University of Lodz, Poland \\ ${ }^{5}$ Histopathology Unit, Olympus Consilio Company Itd, Lodz, Poland \\ ${ }^{6}$ Department of Gynecologic Oncology, Copernicus Memorial Hospital of Lodz, Poland
}

Submitted: 21 August 2007

Accepted: 17 January 2009

Arch Med Sci 2012; 8, 3: 578-581

DOI: $10.5114 /$ aoms.2012.29414

Copyright $\odot 2012$ Termedia \& Banach

Carcinoma of the uterine cervix is the most common gynecologic malignant neoplasm all over the world [1, 2], but the second one in Poland after carcinoma of the endometrium [3]. In 2009 in Poland the number of its new cases was 3102, and the age-adjusted incidence rate was 10.2 per 100000 women. In the age group 20-24 year the age-adjusted incidence rate of the cervical cancer was 0.4 per 100000 , and there were 6 new cases noted in Poland. It constituted $1.86 \%$ of the cancer incidence rate of all localizations in this age group in Poland [3]. The most common histological type of malignant cervical neoplasms is squamous cell carcinoma. Adenocarcinomas account only for approximately $15 \%$ of malignant cervical tumors $[1,2,4,5]$. The screening based on exfoliative cytology introduced in the 1950s by Papanicolau, followed by colposcopy in appropriate patients, is an effective method for identifying squamous intraepithelial lesions [1, 2].

The goals of colposcopy are to identify suspicious areas that require biopsy and to determine the extent of the lesions [2]. Treatment of intraepithelial lesions is determined on the basis of the histological diagnosis and the extent of the lesions on colposcopic examination [1, 2].

Unfortunately, the Pap-smear screening is less efficient, or even of no benefit in cervical adenocarcinomas [4-6]. The marked decrease of the incidence of cervical squamous cell carcinoma in the last decades thanks to the screening appears to be in contrast to the increasing rate of cervical adenocarcinomas [4, 5]. The diagnosis, when cervical cancer does not arise from the squamous epithelium, and additionally the patient is a young woman, can be very difficult.

A 24-year-old woman, without any coexisting diseases, with negative familial cancer history, was admitted to our department in February 2005 because of primary clear cell adenocarcinoma of the uterine cervix. She had reported abnormal vaginal bleeding, non-specific symptoms such as head-ache and nausea, and weight loss (nearly 12 pounds), which had been appearing for the last 6 months. For that time she had been taking

\section{Corresponding author:} Leszek Gottwald MD, PhD Department of Palliative Medicine

Copernicus Memorial Hospital 2 Ciolkowskiego 93-509 Lodz, Poland Phone: +48426895481 Fax: +48 426895482 E-mail: lgottwald@wp.pl 
low doses of oral contraception. She had a history of numerous consultations with an internist, gynecologist, and psychiatrist as well, because of the symptoms described above.

In December 2004, during gynecological examination, a cervical lesion $1.8 \mathrm{~cm} \times 1.2 \mathrm{~cm} \times 1.0 \mathrm{~cm}$ in size was diagnosed, and the Pap-smear was taken. The lesion was described as soft, fragile and hypertrophic. The cytological specimen was classified according to the Bethesda score as adequate for the assessment, with the presence of atypical glandular cells of undetermined significance (AGUS), and it corresponded to Pap III. A biopsy was recommended. The histopathological specimen taken from the uterine cervix showed polypoid tissues with inflammatory changes, glandular hypertrophy and the Arias-Stella reaction. The glandular cells showed signs of macronucleosis and little proliferative activity (measured by Ki-67). Diagnostics to check for endocervical pathology or ectopic pregnancy were recommended. The patient was referred to the gynecological unit with suspected ectopic pregnancy. She was not admitted to the hospital because of lack of clinical, sonographic and hormonal symptoms (low serum $\beta$-human chorionic gonadotropin [ $\beta-\mathrm{HCG}]$ concentration) of ectopic pregnancy.

The patient was hospitalized after 2 weeks in the gynecological unit of another hospital with suspected ectopic pregnancy. In gynecological examination the presence of the cervical lesion was confirmed, and again a Pap-smear and a biopsy were taken. Other pathological findings were not observed. The serum concentrations of $\beta-\mathrm{HCG}$ on consecutive days were all normal $(<0.1 \mathrm{mIU} / \mathrm{ml})$. The sonographic examination showed the uterus with regular shape, sized $43 \mathrm{~mm} \times 31 \mathrm{~mm} \times 46 \mathrm{~mm}$, with the endometrium of $10 \mathrm{~mm}$ thickness. No pathological findings in both adnexa were found. In the sonographic examination, a layer of fluid in the recto-uterine pouch of thickness $11 \mathrm{~mm}$ was visualized. She was discharged from the hospital. The cytological specimen was classified according to the Bethesda system as adequate for the assessment, but limited by inflammation and the numerous metaplastic cells, and it corresponded to Pap II. In the biopsy taken from the cervical erosion clear cell adenocarcinoma stage $\mathrm{lb}$ according to the FIGO classification was diagnosed, and the patient was sent to the oncological unit.

On admission to the Department of Gynecologic Oncology, the concentrations of tumor markers in blood were in the normal range, and their values were as follows: $\mathrm{AgSCC}=5.0 \mathrm{ng} / \mathrm{ml} ; \beta-\mathrm{HCG} \leq 0.1 \mathrm{mlU} / \mathrm{ml}$; $\mathrm{AFP}=1.6 \mathrm{IU} / \mathrm{ml}$; CEA = $1.19 \mathrm{ng} / \mathrm{ml}$; CA $125=20.98$ $\mathrm{IU} / \mathrm{ml}$; CA $19.9 \leq 0.6 \mathrm{IU} / \mathrm{ml}$; CA $72.4=0.826 \mathrm{IU} / \mathrm{ml}$; CA $15.3=18.41 \mathrm{IU} / \mathrm{ml}$; CYFRA $21.1=0.464 \mathrm{ng} / \mathrm{ml}$. The laboratory blood tests, including morphology,
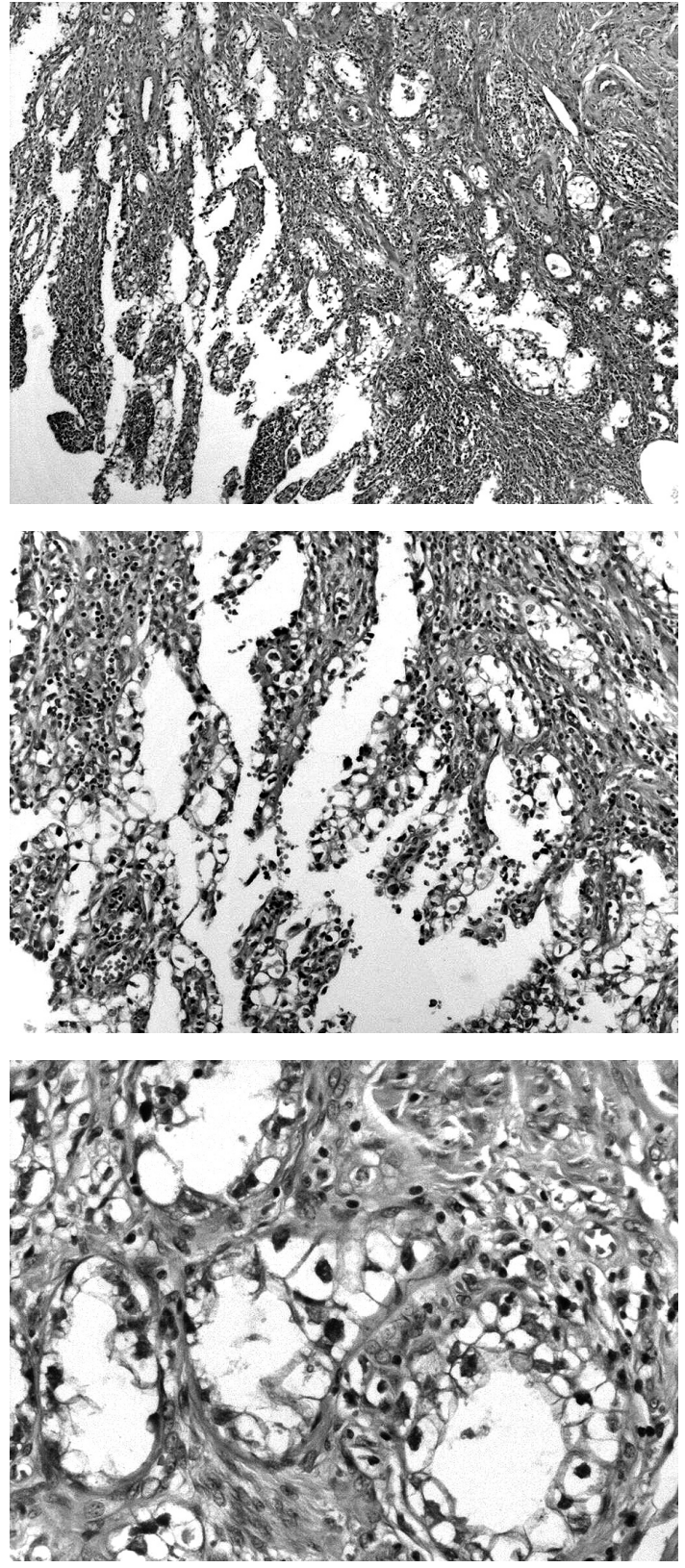

Figure 1. Clear cell adenocarcinoma of the uterine cervix. Hematoxylin and eosin stain, original magnification 40× (A), original magnification 100× (B), original magnification $200 \times$ (C)

electrolytes, creatinine, urea, serum proteins and total bilirubin, presented normal values. The patient was scheduled for a laparotomy. During the operation total hysterectomy with bilateral salpingooophorectomy, appendectomy and radical pelvic lymphadenectomy was performed. No signs of extra-uterine spread of the neoplastic disease was macroscopically observed. Histologically the initial diagnosis of primary cervical clear cell carcinoma stage Ib was confirmed. The postoperative period was uncomplicated, and the patient was discharged from the hospital. After several weeks she was qualified for successive adjuvant radiation therapy 
(teletherapy and brachytherapy). The patient, 88 months after the surgical treatment, is still under observation in our department. No signs of recurrence have been detected since then.

The incidence of malignant neoplasms of the genital organs in young women under 25 years old is relatively low $[2,3]$. The presence of non-specific symptoms, as in our patient, makes the diagnosis more difficult. Additionally, when the cancer is of a rare localization, the popular diagnostic methods could be less effective [4]. These factors together could delay the correct diagnosis and worsen the prognosis for patients [5]. Additionally, we have to emphasize the negative familiar history of cancer, and the lack of epidemiological risk factors of cervical cancer, such as HPV infection, multiple sexual partners, smoking and low socioeconomic status, in the presented case $[1,5]$. No history of exposure to diethylstilbestrol (DES) was noted as well, although it is known that clear cell adenocarcinoma is rare in women without in utero DES exposure and in such cases it concerns in most cases postmenopausal women [7]. The only risk factor present in our patient, but typical for this age group in the population, was the history of oral contraceptive use, which was suggested by some authors to increase the incidence of cervical adenocarcinoma, especially in the group of recent and current users $[4,5]$. The only presented clinical manifestations of the pathological transformation on the uterine cervix in our patient were abnormal vaginal bleeding, according to many authors the most common symptom of cervical adenocarcinoma, and the presence of cervical erosion $[6,8]$.

It is important to note the presence of atypical glandular cells of AGUS in the cytological smear, and the Arias-Stella reaction in the cervical biopsy. It is known that designation of AGUS applies to glandular cells which demonstrate changes beyond those encountered in benign reactive processes, yet which are insufficient for a diagnosis of a preinvasive or invasive glandular neoplasm [1]. Only a minority of women with such findings are at risk for significant lesions, and many of them are evaluated and treated unnecessarily.

The coexistence of AGUS in the smear with the Arias-Stella reaction might be a sign of developing cervical glandular pathology $[9,10]$. The Arias-Stella effect is known as a benign, proliferative change, which occurs in the Mullerian epithelium in response to hyperprogestational status. The reaction was first described by Javier Arias-Stella in 1954 as striking cellular and nuclear atypia of endometrial secretory glands $[9,10]$. These changes can typically occur with ectopic as well as intrauterine pregnancies, but it has been described involving endometriosis and various sites in addition to the endometrium, including the endocervical glands and polyps, vaginal adenosis, paratubal and ovarian cysts, and fallopian tube epithelium [9]. When the effect occurs in an unusual localization such as the cervix, the nature of the changes may not be attributed to the Arias-Stella reaction, and the possibility of a malignant process, especially with the presence of clear cell adenocarcinoma, is often raised. It concerns both the cytological and histological specimen $[9,10]$. According to Nucci and Young [9], as well as Yates et al. [10], despite the large similarities between the cytological appearance of clear cell adenocarcinoma and the AriasStella changes, in most cases two important distinguishing features can be seen. The first is the frequent presence of intranuclear inclusions in cases of Arias-Stella change, which has not been described in clear cell adenocarcinoma. The second is the lack of prominent eosinophilic nucleoli in the Arias-Stella change, which is characteristically connected to cervical clear cell adenocarcinoma $[9,10]$.

According to Shizuko et al., cytological findings of clear cell adenocarcinomas with a predominantly papillary growth pattern are characterized by 1) amorphous substances (substances in the basement membrane) pale green in color at the center of the cluster and 2) a mirror ball or a rod-like cluster and a floret-like small cluster. Therefore, in those cases where the cytological characteristics of clear cell adenocarcinoma are observed, it is possible to predict the histological diagnosis of clear cell adenocarcinoma in the uterine cervix [11]. These signs of the developing cancer are not always seen, unfortunately. In these cases the differential diagnosis can be difficult. Laboratory blood tests for tumor markers, as well as imaging examinations, are only of limited value, as was confirmed in our patient, and a biopsy in each case has to be done.

In the described case, the first biopsy, which was taken from the cervical erosion, did not show any signs of malignancy, while the second one showed the presence of cancer. Both biopsies were taken unfortunately without the use of colposcopy. We hold the opinion that in each suspected feature which concerns the uterine cervix, even in young women, biopsies during colposcopic assessment of the uterine cervix should be taken.

The data from the literature indicate that in primary clear cell adenocarcinoma, either radiation therapy or radical hysterectomy and bilateral lymph node dissection, by an experienced professional, results in cure rates of $85 \%$ to $90 \%$ for patients with small volume disease [4]. The mode of treatment depends on patient factors and available local experience. The size of the primary tumor is an important prognostic factor and should be carefully evaluated in choosing optimal therapy [2, 8]. For adenocarcinomas that expand the cervix greater than $3 \mathrm{~cm}$, the primary treatment should be radia- 
tion therapy. In smaller foci, as in our patient, initial surgery followed by radiation therapy should be performed [2, 6]. The American Brachytherapy Society has published guidelines for the use of low-dose rate (LDR) and high-dose rate (HDR) brachytherapy as components of cervical cancer treatment [12].

It is recommended in surgical treatment of cervical cancer that if the depth of invasion is less than $3 \mathrm{~mm}$, no vascular or lymphatic channel invasion is noted, and the margins of the cone are negative, conization alone may be appropriate in patients wishing to preserve fertility [13]. Unfortunately in the present case because of the higher local advanced disease, the radical hysterectomy was done. The U.S. National Cancer Institute recommends for patients with cervical cancer stage IA and tumor invasion more than $3 \mathrm{~mm}$, as well as cervical cancer stage IB, radical hysterectomy with pelvic node dissection [13].

Survival of patients with cervical adenocarcinoma was recently reported in five randomized phase III trials to be improved when combination of postoperative radiation and platinum-based chemotherapy is applied [4, 8, 14, 15], while one trial examining this regimen demonstrated no benefit [16]. The mode of treatment depends in most cases on the experience of the oncology center. In the Regional Center for Oncology in Lodz in cervical adenocarcinoma stage IB according to the FIGO classification, only radiation therapy following radical hysterectomy is a treatment of choice.

The question whether cervical clear cell adenocarcinoma and adenocarcinoma have worse prognosis than squamous cell carcinoma of the uterine cervix remains open $[4,17,18]$. Korhonen suggested, after the analysis of 163 cases of primary cervical adenocarcinomas of different subtypes, that the prognosis of clear cell carcinomas is similar to that of non-clear cell cervical adenocarcinomas [17]. Reich et al. did not find a statistically significant difference in the prognosis of surgically treated patients with stage IB-IIB clear cell carcinomas, squamous cell carcinomas and non-clear cell adenocarcinomas [18]. However, Niibe et al., based on a literature review, suggested that the 5-year survival rate in cervical adenocarcinoma is worse than the 5-year survival rate in cervical squamous cell carcinoma [14]. A similar opinion was presented by Quinn and Freitag et al. [4, 6]. All authors agree that among the major factors that influence prognosis of each histopathological type of cervical cancer, the most important is the stage of the disease $[1,2$, $4,6,14,18]$.

\section{References}

1. Cannistra S, Niloff J. Cancer of the uterine cervix. N Engl J Med 1996; 334: 1030-7.

2. Benedet JL, Bender $\mathrm{H}$, Jones $\mathrm{H}$. Staging classifications and clinical practice guidelines of gynaecologic cancers. FIGO
Committe on Gynecologic Oncology. Elsevier, 2000; 36-58.

3. Department of Epidemiology and Cancer Prevention. Maria Sklodowska-Curie Institute of Warsaw. Polish National Cancer Registry, Cancer in Poland in 2009. http://www.onkologia.org.pl.

4. Quinn MA. Adenocarcinoma of the cervix. An Acad Med Singapore 1998; 27: 662-5.

5. Liu S, Semenciw R, Mao Y. Cervical cancer: the increasing incidence of adenocarcinoma and adenosquamous carcinoma in younger women. CMAJ 2001; 164: 1151-2.

6. Freitag P, Macku J, Nouzova K. Adenocarcinoma of the uterine cervix: retrospective clinico-pathologic study. Ceska Gynekol 1996; 61: 162-4.

7. Schrager S, Potter BE. Diethylstilbestrol exposure. Am Fam Physician 2004; 69: 2395-400.

8. Seki H, Takada T, Sodemoto T, Hoshino H, Saitoh K, Uekusa T. A young woman with clear cell adenocarcinoma of the uterine cervix. Int J Clin Oncol 2003; 8: 399-404.

9. Nucci MR, Young RH. Arias-Stella reaction of the endocervix: a report of 18 cases with emphasis on its varied histology and differential diagnosis. Am J Surg Pathol 2004; 28: 608-12.

10. Yates WA, Persad RV, Standbridge CM. The Arias-Stella reaction in the cervix: a case report with cervical cytology. Cytopathology 1997; 8: 40-4.

11. Shizuko K, Takashi K, Masaaki J, et al. A case of clear celladenocarcinoma with a predominantly papillary growth pattern in the uterine cervix. J Jap Soc Clin Cytol 2004; 43: 166-70.

12. Nag S, Chao C, Erickson B, et al. The American Brachytherapy Society recommendations for low-dose-rate brachytherapy for carcinoma of the cervix. Int J Radiat Oncol Biol Phys 2002; 52: 33-48.

13 National Cancer Institute. U.S. National Institutes of Health. Cervical Cancer: treatment. http://www.cancer.gov/ cancertopics/pdq/treatment/carvical/HealthProfessional/ page1-7

14. Niibe $\mathrm{Y}$, Karasawa K, Kaizu T, et al. Difference in prognostic factors between stage Ib and II uterine cervical carcinoma patients treated with radical hysterectomy and postoperative radiation therapy. Radiat Med 2002; 20: 161-7.

15. Thomas GM. Improved treatment for cervical cancer; concurrent chemotherapy and radiotherapy. N Engl J Med 1999; 340: 1198-200.

16. Pearcey R, Brundage M, Drouin P, et al. Phase III trial comparing radical radiotherapy with and without cisplatin chemotherapy in patients with advanced squamous cell cancer of the cervix. J Clin Oncol 2002; 20: 966-72.

17. Korhonen MO. Adenocarcinoma of the uterine cervix. Prognosis and prognostic significance of histology. Cancer 1984; 53: 1760-3.

18. Reich O, Tamussino K, Lahousen M, Pickel H, Haas J, Winter R. Clear cell carcinoma of the uterine cervix: pathology and prognosis in surgically treated stage IB-IIB disease in women not exposed in utero to diethylstilbestrol. Gynecol Oncol 2000; 76: 331-5. 